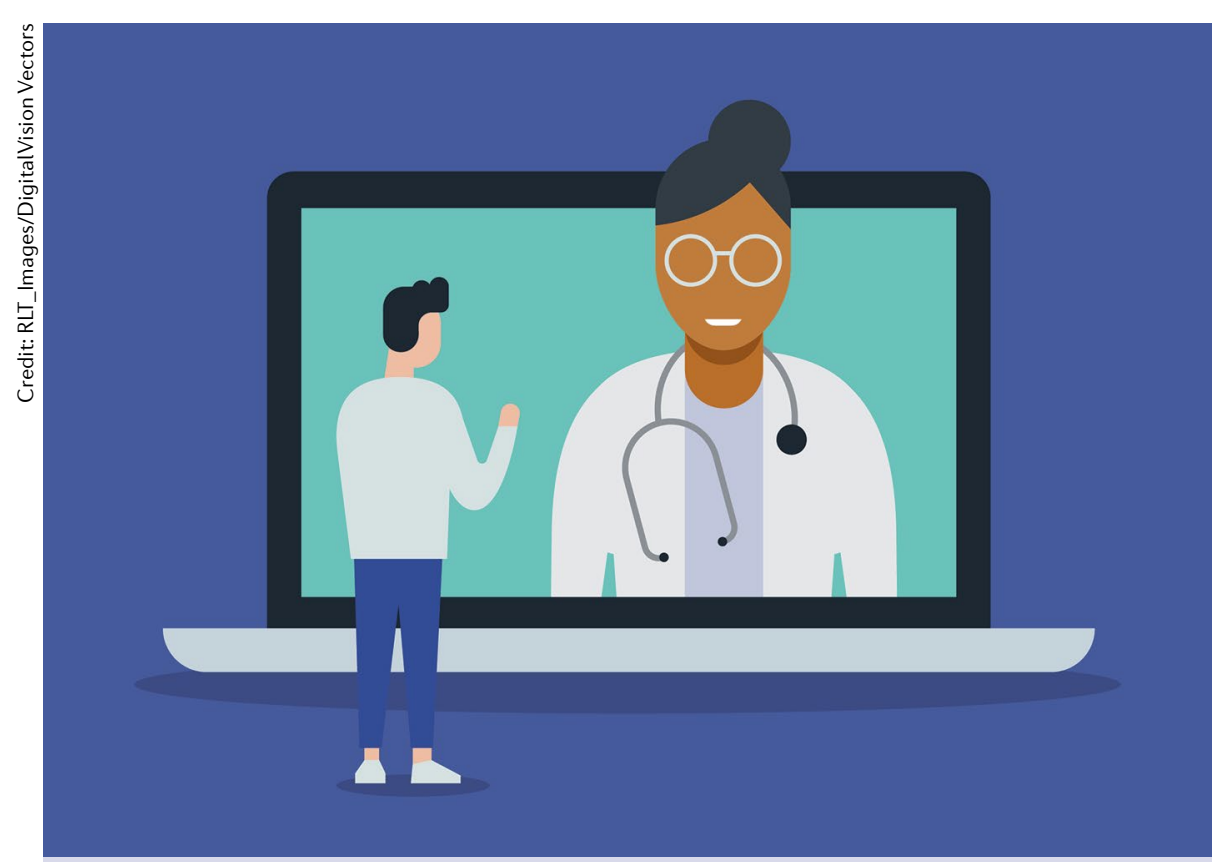

RHEUMATOID ARTHRITIS

\title{
Adopting PROs in virtual and outpatient management of RA
}

\author{
Peter C. Taylor (iD \\ The COVID-19 pandemic has catalysed the sudden adoption of \\ telemedicine in the management of rheumatic diseases. In this abrupt \\ transition from in-person visits to telemedicine, can patient-reported \\ outcomes (PROs) help ensure that we continue to achieve optimum \\ disease control and address the concerns of people living with \\ rheumatoid arthritis?
}

Refers to Mistry, J. et al. Use of rheumatoid arthritis impact of disease (RAID) in routine care; identification of DAS28 remission and unmet patient reported outcomes. Rheumatol. Adv. Pract. https://doi.org/10.1093/rap/rkaa013 (2020).

The sudden emergence of SARS-CoV-2 onto the world stage, and the high morbidity and mortality associated with COVID-19 symptoms in a proportion of those infected, has accelerated a major change in the management of patients with rheumatic diseases and, in particular, has catalysed the rapid emergence of telemedicine. However, judging which patients might be most suited to a remote consultation is not a straightforward matter. Findings from a new study suggest that the Rheumatoid Arthritis Impact of Disease (RAID) score could help identify the patients with rheumatoid arthritis (RA) who are best suited to a telemedicine consultation and could also promote holistic management of subjective symptoms that might otherwise be overlooked ${ }^{1}$.

The ideal immunological goal of RA management is to correct the immune dysregulation that characterizes the disease and causes joint destruction and systemic comorbidity and to restore immune homeostasis. In doing so, the hope is that the subjective symptomatology accompanying RA will correspondingly resolve. But in the absence of biomarkers that reliably inform management decisions, the accepted paradigm is to treat to a target of remission or low disease activity, as assessed by composite scores of disease activity, and to titrate therapy according to the response. However, in the context of a pandemic, rheumatologists must be mindful that many of the pharmacological interventions in the now substantial armamentarium are potentially immunosuppressive and might therefore increase the risk of viral contagion. Conversely, it has emerged that the pathobiology of COVID-19 mortality is related to exaggerated host immune responses, and for this reason, several rheumatology drugs that target cytokines are being investigated as potential treatments in people with severe COVID-19 related pathology ${ }^{2}$.

In the face of the COVID-19 pandemic, rheumatologists have stratified the risk of infection in people with RA according to their treatment regime, age and comorbidities. But at the same time, the importance of maintaining 'tight control' of inflammation has been emphasized with a view to minimizing the risks associated with inadequately treated RA and in the knowledge that poorly controlled disease is in itself a risk factor for serious infectious complications ${ }^{3}$. Abundant evidence from many clinical studies support the effectiveness of a continuous goal-oriented treatto-target approach, with respect to improved and maintained clinical, functional and radiographic outcomes ${ }^{4}$. At a group level, patientreported outcomes (PROs) also improve. However, the aspirational target of sustained remission or, failing that, low disease activity, is not attainable for many individuals. Furthermore, among those patients who do achieve the therapeutic target, subjective symptoms can remain. Prominent among these symptoms are pain, fatigue and functional loss ${ }^{5}$. As these symptoms are known only to the patient themselves, they might be overlooked when treating to a disease activity target, particularly in the context of a truncated consultation during a pandemic.

The use of PROs could be one method of deciding which patients would be suited to a telehealth consultation while ensuring that disease activity is controlled and symptoms important to the patient aren't missed. In the new study, Mistry et al. ${ }^{1}$ assessed the performance of the RAID score relative to the 28-Joint Disease Activity Score (DAS28) in a cohort of 198 people with established RA. RAID was developed as a EULAR initiative to combine the most important PROs into one measure ${ }^{6}$. This tool comprises seven domains encompassing pain, fatigue, physical function, sleep, physical and emotional well-being and coping. Each domain is scored using a numeric rating scale, giving a total score of $0-10$, with higher scores indicating greater disease impact. A RAID score of $<2$ is regarded as a Patient Acceptable Symptom State $(\mathrm{PASS})^{1}$, that is, a symptom state that the patient considers acceptable. The PASS stems from the concept that feeling good is more

poorly controlled disease is in itself a risk factor for serious infectious complications 
important to patients than feeling better ${ }^{7}$. The researchers of the new study noted that the patients reported no difficulties in understanding or completing the RAID questions, which can be done in advance of the consultation and can take less than 5 minutes. Rapidness and ease of use is an important consideration for those rheumatologists working in health-care systems where contact time with the patient is constrained. Furthermore, the fact that a patient can complete the RAID questions prior to either an in-person or telehealth appointment might reduce consultation time by highlighting the health issues of most importance to the individual.

\section{$\int$ the good physician treats the disease, but the great physician treats the patient who has the disease}

The RAID scores correlated strongly with the DAS28 when calculated with the erythrocyte sedimentation rate (DAS28-ESR) or the C-reactive protein concentration (DAS28-CRP) and also correlated with the patient global assessment (PGA) component of the DAS28 composite score ${ }^{1}$. Notably, the PGA covers two concepts - global health and overall disease activity - and is the PRO incorporated into most of the ACR-endorsed and EULARendorsed RA assessment tools. Although the PGA is included in the DAS28, its weighting is low, and so it has relatively little influence on the final DAS28 score. Furthermore, PGA might be influenced by many factors including pain, mood and fatigue. In contrast to the DAS28, the RAID sheds light on which subjective symptoms most affect the patient and so might inform on which management plan is most appropriate.

The relationship between each subcomponent of the DAS28 and the RAID score was explored using mixed-effects linear regression. Mistry et al. ${ }^{1}$ found that both the patient global visual analogue scale and the square root tender joint count were strongly associated with RAID scores $(P<0.01)$. Importantly, of the 66 patients with a RAID score $<2,92-97 \%$ met remission criteria, and $98.5 \%$ were in remission or low disease activity according to DAS28-ESR or DAS28-CRP thresholds. These data give considerable confidence that in a telemedicine setting, patients reporting a RAID score of $<2$ would have attained a DAS28 treat-to-target goal. Conversely, of the 134 patients in low disease activity or remission, $51.5 \%$ had a RAID score $\geq 2$. This high proportion of patients with symptomatology in the unacceptable range, despite meeting contemporary disease activity treatment targets, cautions against over-reliance on protocoldriven medicine in the outpatient clinic and emphasises the value of PROs used adjunctively to composite scores of disease activity $^{8}$. The RAID domains that had the largest proportion of individuals with a score in the severe range (7-10) were fatigue (35.6\%), sleep (33.3\%) and emotional well-being (28.9\%). In the context of outpatient clinic consultations, inspection of scores across the RAID domains might help to quickly identify important unmet needs for an individual who has attained the disease activity treatment target. In doing so, the physician can initiate beneficial, non-pharmacological management approaches such as lifestyle advice and cognitive behavioural therapy, both of which could be amenable to telemedicine delivery.

A good therapeutic goal from the perspective of a patient is 'to have a good day'. The evidence suggests that RAID can assist in shared decision making, whether in an outpatient clinic or telemedicine setting, to achieve this goal. RAID could also be used to triage those patients best suited to telemedicine follow up. As expressed so well by Sir William
Osler, rheumatologists should be mindful that "the good physician treats the disease, but the great physician treats the patient who has the disease" ${ }^{10}$.

Peter C. Taylor iD

Botnar Research Centre, Nuffield Department of Orthopaedics, Rheumatology and Musculoskeletal Sciences, University of Oxford, Oxford, UK. e-mail:peter.taylor@kennedy.ox.ac.uk https://doi.org/10.1038/s41584-020-0449-6

1. Mistry, J. et al. Use of rheumatoid arthritis impact of disease (RAID) in routine care; identification of DAS28 remission and unmet patient reported outcomes. Rheumatol. Adv. Pract. https://doi. org/10.1093/rap/rkaa013 (2020).

2. Mehta, P. et al. COVID-19: consider cytokine storm syndromes and immunosuppression. Lancet 395 1033-1034 (2020).

3. Wolfe, F. et al. The mortality of rheumatoid arthritis. Arthritis Rheum. 37, 481-94 (1994).

4. Stoffer, M. A. et al. Evidence for treating rheumatoid arthritis to target: results of a systematic literature search update. Ann. Rheum. Dis. 75, 16-22 (2016).

5. Taylor, P. C., Moore, A., Vasilescu, R., Alvir, J. \& Tarallo, M. A structured literature review of the burden of illness and unmet needs in patients with rheumatoid arthritis: a current perspective. Rheumatol. Int. 36, 685-695 (2016).

6. Gossec, L. et al. Elaboration of the preliminary Rheumatoid Arthritis Impact of Disease (RAID) score: a EULAR initiative. Ann. Rheum. Dis. 68, 1680-1685 (2009).

7. Tubach, F. et al. Minimal clinically important improvement and patient acceptable symptom state for subjective outcome measures in rheumatic disorders. J. Rheumatol. 34, 1188-1193 (2007).

8. Fautrel, B. et al. Call for action: how to improve use of patient-reported outcomes to guide clinical decision making in rheumatoid arthritis. Rheumatol. Int. 38, 935-947 (2018)

9. Strand, V., Wright, G., Bergman, M., Tambiah, J. \& Taylor, P. C. Patient expectations and perceptions of goal-setting strategies for disease management in rheumatoid arthritis. J. Rheumatol. 42, 2046-2054 (2015).

10. Taylor, P. C. \& Pope, J. Treating to target or treating the patient in rheumatoid arthritis? Lancet Rheumatol. 1, e8-e10 (2019).

\section{Acknowledgements}

P.T. thanks the National Institute of Health Research for their funding of The NIHR Biomedical Research Centre in Musculoskeletal Disease at Oxford University Hospitals NHS Trust and the University of Oxford.

\section{Competing interests}

The author declares no competing interests. 